Review

\title{
The Use of Probiotic Strains in Caries Prevention: A Systematic Review
}

\author{
Maria Grazia Cagetti ${ }^{1}$, Stefano Mastroberardino ${ }^{1}$, Egle Milia ${ }^{2}$, Fabio Cocco ${ }^{3}$, \\ Peter Lingström ${ }^{4}$ and Guglielmo Campus ${ }^{1,2, *}$
}

1 WHO Collaborating Centre of Milan for Epidemiology and Community Dentistry, Department of Health Sciences, University of Milan, Milan 20142, Italy;

E-Mails: maria.cagetti@unimi.it (M.G.C.); stefano.mastroberardino@hotmail.it (S.M.)

2 Department of Surgery, Microsurgery and Medical Sciences, School of Dentistry, University of Sassari, Sassari 07100, Italy; E-Mail: emilia@uniss.it

3 Department of Chemistry and Pharmacy, University of Sassari, Sassari 07100, Italy; E-Mail: fcocco@uniss.it

4 Department of Cariology, Institute of Odontology, The Sahlgrenska Academy, University of Gothenburg, Gothenburg SE-405 30, Sweden; E-Mail: peter.lingstrom@odontologi.gu.se

* Author to whom correspondence should be addressed; E-Mail: gcampus@uniss.it; Tel.: +39-079-228540; Fax: +39-079-228541.

Received: 4 June 2013; in revised form: 13 June 2013 / Accepted: 17 June 2013 /

Published: 5 July 2013

\begin{abstract}
This paper aims to provide a systematic review of the caries-prevention effect of probiotics in human. The hypothesis was that the administration of probiotic strains might play a role in caries lesion prevention and in the control of caries-related risk factors. The main relevant databases (Medline, Embase) were searched. Quality of the Randomized Clinical Trials (RCTs) was classified using the "Consolidated Standards of Reporting Trials" (CONSORT) checklist and the Impact Factor (IF) value of each journal was recorded. Sixty-six papers were identified, and 23 fulfilled the inclusion criteria. Only three studies had caries lesion development as outcome, all the others reported caries risk factors as interim evaluation. Using the CONSORT Score, the papers were coded as 4 excellent, 9 good and 10 poor. The mean IF value recorded was 1.438 . Probiotics may play a role as antagonistic agent on mutans streptococci (MS), acidogenic/aciduric bacteria that contributes to the caries process. In two-thirds of the selected papers, probiotics have demonstrated the capacity to reduce MS counts in saliva and/or plaque in short-term. The effect of probiotics
\end{abstract}


on the development of caries lesion seems encouraging, but to date, RCTs on this topic are insufficient to provide scientific clinical evidence.

Key words: probiotics; dental caries; dental caries prevention; cariogenic bacteria; mutans streptococci; lactobacilli; plaque $\mathrm{pH}$; plaque acidogenicity

\section{Introduction}

Dental caries still remains one of the most common diseases worldwide, although a decline of the prevalence has been recorded in western countries [1-3]. The disease is triggered by the interaction over time among cariogenic microorganisms (mainly mutans streptococci and lactobacilli), a diet rich in fermentable carbohydrates and host factors, like as saliva secretion rate and buffering capacity [4]. Mutans streptococci (MS) have been considered for a long time the major pathogens involved in caries development. Nevertheless, in recent years, it was described that the microflora on the tooth surface changes with caries lesion development, from a predominance of non-mutans streptococci and Actinomyces spp. to dominance of MS and other non-mutans bacteria, including lactobacilli and Bifidobacterium spp. [5].

When sugared food/drinks are supplied frequently, acidogenic and aciduric strains increase selectively in the oral environment. These changes, over time, shift the demineralization/remineralization balance toward net mineral loss, leading to the caries lesion development [6]. Preventive strategies are needed and recommended to control caries risk factors mainly based on dietary changes i.e., sweeteners intake reduction and enhancing host resistance [7,8]. Sometimes, antibacterial agents are administered in order to reduce cariogenic micro-flora, however, a complete eradication of caries-associated microorganisms has proved to be difficult and almost impossible to obtain [9].

The World Health Organization has defined probiotics as "Live microorganisms which, when administered in adequate amounts, confer a health benefit on the host" [10]. These microorganisms belong to the natural human flora in order to survive in the acid environment during transit to the intestines.

Probiotics are recognized to perform several actions in the digestive system as to prevent cellular adhesion and invasion of pathogenic bacteria, modify the intestinal environment and modulating the local and systemic inflammatory immune response [11].

Recent reviews have reported on the use of probiotic strains for the prevention of oral diseases, including caries $[12,13]$. Probiotics are administered to maintain or restore the natural saprophytic micro-flora against a pathogen invasion, which is central to the development of the major oral diseases (caries and periodontal disease). Probiotic strains administered for oral care are microorganisms mainly used to obtain gastrointestinal benefits, so they might not be ideal for the oral environment, quite different from the intestinal habitat. The effect of probiotics on dental caries and its related risk factors has been evaluated in several experimental studies [14-36], using different strains; Lactobacillus rhamnosus GG, L. casei, L. reuteri, L. plantarum, L. brevis CD2, Bifidobacterium spp. etc. were proposed and used to obtain caries incidence reduction, mutans streptococci and lactobacilli count change, plaque $\mathrm{pH}$ control and root caries lesions reversal. 
Several appropriate vehicles of administration of probiotic strains have been proposed. Dairy products supplemented with probiotics are a natural means of oral administration and easily adopted in dietary regime for adults and children. However, specifically formulated devices with slow release of the microbial strain might be needed in order to oral diseases prevention and control.

Another uncertain aspect of the probiotic use is whether the probiotics species really are able to colonize the oral habitat, and how long the microbial shift was induced [37]. It is well established for probiotics in the gastrointestinal tract that they usually colonize for a short time only [38]. Therefore, a prolonged administration of the probiotics bacteria seemed is mandatory to improve the benefits of the treatment.

The hypothesis behind this systematic review was that the administration of probiotic strains might play a role in the caries lesion prevention and in the control of caries-related risk factors.

\section{Materials and Methods}

\subsection{Eligibility Criteria}

The studies included in the present review are Randomized Clinical Trials assessing the in vivo role of probiotics administration on caries lesion development and on caries risk factors control (cariogenic micro-flora, plaque $\mathrm{pH}$, etc.). Only human studies considering subjects without any stated medical condition were considered. Only studies in English were collected, due to the virtual absence of research published in other languages as a result of preliminary electronic database searches. All in vitro studies, all studies not focusing on probiotics administration for caries prevention and studies where probiotics were administered for other reasons were excluded.

\subsection{Search Strategy}

The main important electronic databases were searched: Medline from 01 January 1966 to 15 May 2013 and Embase from 1973 to 15 May 2013. Two preliminary searches were conducted in March 2013 in order to obtain an overall idea of findings and to polish search terms (MeSH words) and limits. The MeSH Browser was accessed to identify entry terms and compose the final Boolean searches [39].

The first step was the association of MeSH terms Dental Caries and Probiotic(s); after that, a combination of key words derived from the two previous MeSH terms were searched for a total of 18 inquiries. The key words used were: Caries, Probiotic Bacteria, Probiotic Lactobacilli, Bacteriotherapy, Dental Disease, Oral Health, Oral Streptococci, Cariogenic bacteria, Plaque pH and Dental Caries Susceptibility.

A comparison of the 18 different searches was carried out to delete the repeated studies. Then, two authors (M.G.C. and S.M.) examined independently all abstracts of the selected papers. All studies, which appeared to meet the inclusion criteria, were obtained in the full text format. The two authors assessed the papers independently, to establish whether or not the studies met the inclusion criteria.

Disagreements were resolved by discussion. If not possible, other authors were consulted. All studies meeting the inclusion criteria then went to a validity assessment. Studies rejected at this or subsequent stages are reported in the Table 1 of excluded studies with the reasons for exclusion [40-82]. For each trial, the following information was recorded: citation details; participants: including 
demographic characteristics and criteria for inclusion; intervention: including type and duration of intervention, duration of follow-up and method of administration.

Table 1. List of papers not included in the review.

\begin{tabular}{|c|c|}
\hline List of excluded studies & $\begin{array}{l}\text { Reasons for exclusion } \\
\text { (all different outcome) }\end{array}$ \\
\hline Keller et al. [40] & Oral malodour \\
\hline Wang et al. [41] & Intestinal health \\
\hline Allen et al. [42] & Diarrhoea \\
\hline Iniesta et al. [43] & Gingival health \\
\hline Slawik et al. [44] & Gingival health \\
\hline Vandenplas et al. [45] & Acute gastroenteritis \\
\hline Burton et al. [46] & Safety and tolerance \\
\hline Krauss-Silva et al. [47] & Preterm delivery \\
\hline Hummelen et al. [48] & Human immunodeficiency virus (HIV) \\
\hline Harini et al. [49] & Gingival health \\
\hline Saxelin et al. [50] & Gastrointestinal persistence \\
\hline Hummelen et al. [51] & Bacterial vaginosis \\
\hline Arroyo et al. [52] & Infectious mastitis \\
\hline Grossi et al. [53] & Diarrhoea \\
\hline Sierra et al. $[54]$ & Intestinal effect \\
\hline Sinkiewicz et al. [55] & Gingival health \\
\hline Mayanagi et al. [56] & Gingival health \\
\hline Dommels et al. [57] & Intestinal persistence \\
\hline Ranganathan et al. [58] & Kidney disease \\
\hline Twetman et al. [59] & Gingival health \\
\hline Basu et al. [60] & Diarrhoea \\
\hline Staab et al. [61] & Gingival health \\
\hline Mao et al. [62] & Diarrhoea \\
\hline Shimauchi et al. [63] & Gingival health \\
\hline Marcone et al. [64] & Bacterial vaginosis \\
\hline Panigrahi et al. [65] & Neonatal gut colonization \\
\hline Mohan et al. [66] & Intestinal health \\
\hline Ivory et al. [67] & Allergic rhinitis \\
\hline Htwe et al. [68] & Diarrhoea \\
\hline Larsson et al. [69] & Bacterial vaginosis \\
\hline Hatakka et al. [70] & Oral candida \\
\hline Basu et al. [71] & Diarrhoea \\
\hline Henker et al. [72] & Diarrhoea \\
\hline Sugawara et al. [73] & Biliary cancer surgery \\
\hline Krasse et al. [74] & Gingival health \\
\hline Margreiter et al. [75] & Diarrhoea \\
\hline Olivares et al. [76] & Intestinal health \\
\hline Sarker et al. [77] & Diarrhoea \\
\hline Schrezenmeir et al. [78] & Acute bacterial infections \\
\hline
\end{tabular}


Table 1. Cont.

\begin{tabular}{cc}
\hline Reid et al. $[79]$ & Bacterial vaginosis \\
Morelli et al. $[80]$ & Vaginal colonization \\
Reid et al. $[81]$ & Vaginal colonization \\
Arvola et al. $[82]$ & Diarrhoea \\
\hline
\end{tabular}

\subsection{Quality Assessment}

The quality of the trials was assessed through the "Consolidated Standards of Reporting Trials" (CONSORT) guidelines [83], using the CONSORT 2010 checklist. The 25 -items checklist is focused on how the trial was designed, analyzed and interpreted. The quality was classified in three categories according to CONSORT score: excellent ( $\geq 20$ items), good (between 13 and 19 items) and poor $(\leq 12$ items) [84].

The Impact Factor, for each journal where the RCTs were published, was determined from ISI Journal Citation Report, 2011 JCR Science Edition [85].

\section{Results}

Sixty-six (66) papers were identified and assessed, and of these, 23 fulfilled the inclusion criteria and they are reported in Tables 2-4 [14-36].

No differences were observed between the two main databases used. Selected papers were divided between those performed on children/adolescents and those on adults. All studies utilized parallel arms with intervention and a placebo/control or a crossover design. The sample sizes were generally small or medium, and the majority of them (80\%) were short-term interventions (between 10 and 42 days). Different vehicles for the administration and different dosage of probiotics were used. The quality of published papers recorded using the Consort Score was: 4 excellent, 9 good and 10 poor. All papers, except two [19,23], were published on Journals with positive IF with a mean value of 1.438 . 
Table 2. Studies with caries risk factors as outcome (children/adolescents).

\begin{tabular}{|c|c|c|c|c|c|c|c|c|}
\hline $\begin{array}{c}\text { Reference } \\
\text { Study design }\end{array}$ & Outcome(s) & Subjects Age & Strain (Concentration) & $\begin{array}{c}\text { Delivery System/ } \\
\text { Treatment Duration }\end{array}$ & Groups & Results & $\begin{array}{c}\text { Consort } \\
\text { score }\end{array}$ & IF score \\
\hline $\begin{array}{c}\text { Taipale et al., } \\
2013[14]\end{array}$ & $\begin{array}{l}\text { MS in plaque } \\
\text { (plate culturing) }\end{array}$ & $\begin{array}{l}106 \text { children } \\
(4 \text { years })\end{array}$ & $\begin{array}{c}\text { Bifidobacterium animalis } \\
\text { subsp. } \\
\text { lactis } \mathrm{BB}-12 \\
\left(10^{10} \mathrm{CFU} / \mathrm{mL}\right)\end{array}$ & $\begin{array}{l}\text { Tablets in slow-release pacifier or } \\
\text { spoon twice daily/22-23 months }\end{array}$ & $\begin{array}{l}\text { A: Probiotic } \\
\text { B: Xylitol } \\
\text { C: Sorbitol }\end{array}$ & $\begin{array}{c}\text { No statistically } \\
\text { significant MS differences } \\
\text { among groups }\end{array}$ & excellent & 2.328 \\
\hline $\begin{array}{l}\text { Campus et al., } \\
2013 \text { [15] }\end{array}$ & $\begin{array}{l}\text { MS in saliva and } \\
\text { plaque } \mathrm{pH} \\
\text { (plate culturing) }\end{array}$ & $\begin{array}{l}191 \text { children } \\
(6-8 \text { years })\end{array}$ & $\begin{array}{l}\text { Lactobacillus brevis } \\
\qquad \mathrm{CD} 2\left(2 \times 10^{9} / \mathrm{g}\right)\end{array}$ & Lozenges twice a day/ 6 weeks & $\begin{array}{l}\text { A: Probiotic } \\
\text { B: Placebo }\end{array}$ & $\begin{array}{c}\text { Statistically significant } \\
\text { decrease in MS and increase } \\
\text { in plaque } \mathrm{pH} \text { in group } \mathrm{A}\end{array}$ & excellent & 2.364 \\
\hline $\begin{array}{c}\text { Juneja et al., } \\
2012[16]\end{array}$ & $\begin{array}{c}\text { MS in saliva } \\
\text { (chair-side tests) }\end{array}$ & $\begin{array}{c}40 \text { children } \\
(12-15 \text { years })\end{array}$ & $\begin{array}{l}\text { Lactobacillus rhamnosus } \\
\text { hct } 70 \\
\left(2.34 \times 10^{9} \mathrm{CFU} / \text { day }\right)\end{array}$ & Milk twice daily/3 weeks & $\begin{array}{c}\text { A: Milk } \\
\text { B: Milk + Probiotic }\end{array}$ & $\begin{array}{l}\text { Statistically significant } \\
\text { reduction in MS immediately } \\
\text { after consumption and after } \\
3 \text { week follow-up in group A }\end{array}$ & poor & 0.444 \\
\hline $\begin{array}{c}\text { Taipale et al., } \\
2012[17]\end{array}$ & $\begin{array}{l}\text { MS in plaque and } \\
\mathrm{Lb} \text { and yeasts in } \\
\text { mucosa/teeth } \\
\text { (plate culturing) }\end{array}$ & $\begin{array}{l}106 \text { infants } \\
(1 \text { month) }\end{array}$ & $\begin{array}{c}\text { Bifidobacterium animalis } \\
\text { subsp. } \\
\text { lactis } \mathrm{BB}-12 \\
\left(10^{10} \mathrm{CFU} / \mathrm{mL}\right)\end{array}$ & $\begin{array}{l}\text { Tablets in slow-release pacifier } \\
\text { or spoon twice daily/months }\end{array}$ & $\begin{array}{l}\text { A: Probiotic } \\
\text { B: Xylitol } \\
\text { C: Sorbitol }\end{array}$ & $\begin{array}{l}\text { MS colonization statistically } \\
\text { significant differ, lactobacilli } \\
\text { and yeasts not differ } \\
\text { among groups }\end{array}$ & excellent & 2.328 \\
\hline $\begin{array}{l}\text { Singh et al., } \\
2011[21] \\
\text { cross-over study }\end{array}$ & $\begin{array}{l}\text { MS and Lb in } \\
\text { saliva } \\
\text { (chair-side tests) }\end{array}$ & $\begin{array}{c}40 \text { children } \\
(12-14 \text { years })\end{array}$ & $\begin{array}{c}\text { Bifidobacterium lactis } \\
\text { Bb-12 ATCC27536 and } \\
\text { Lactobacillus acidophilus } \\
\text { La-5 }\left(10^{6} \mathrm{CFU} / \mathrm{g}\right)\end{array}$ & Ice-cream/10 days & $\begin{array}{c}\text { A: Ice-cream } \\
\text { B: Ice-cream/probiotics }\end{array}$ & $\begin{array}{c}\text { Statistically significant } \\
\text { reduction in MS in group B, } \\
\text { but no significant } \\
\text { effect on lactobacilli }\end{array}$ & good & 1.066 \\
\hline
\end{tabular}


Table 2. Cont.

\begin{tabular}{|c|c|c|c|c|c|c|c|c|}
\hline $\begin{array}{l}\text { Aminabadi et al., } \\
\quad 2011[22]\end{array}$ & $\begin{array}{c}\text { MS in saliva } \\
\text { (plate culturing) }\end{array}$ & $\begin{array}{l}105 \text { children } \\
(6-12 \text { years })\end{array}$ & $\begin{array}{c}\text { Lactobacillus } \\
\text { rhamnosus } \mathrm{GG} \\
\left(2 \times 10^{8} \mathrm{CFU} / \mathrm{mL}\right)\end{array}$ & $\begin{array}{c}\text { Yogurt } / 3 \text { weeks } \\
\text { (chlorhexidine mouthrinse } 2 \text { weeks) }\end{array}$ & $\begin{array}{c}\text { A: Chlorhexidine } \\
\text { B: Probiotic } \\
\text { C: Chlorhexidine, } \\
\text { than probiotic }\end{array}$ & $\begin{array}{l}\text { probiotic use in group B; } \\
\text { recolonization during the } \\
5 \text { consecutive weeks. In } \\
\text { group C a statistically } \\
\text { significant MS reduction that } \\
\text { enhances during the } \\
5 \text { consecutive weeks }\end{array}$ & good & 2.328 \\
\hline $\begin{array}{l}\text { Jindal et al., } \\
2011[23]\end{array}$ & $\begin{array}{c}\text { MS in saliva } \\
\text { (plate culturing) }\end{array}$ & $\begin{array}{l}150 \text { children } \\
(7-14 \text { years })\end{array}$ & $\begin{array}{c}\text { Lactobacillus rhamnosus, } \\
\text { Bifidobacterium longum, } \\
\text { Saccharomyces cereviasae } \\
\text { (1.25 billion) } \\
\text { Bacillus coagulans } \\
\text { (150 million) }\end{array}$ & $\begin{array}{l}\text { Powders (dissolved in water and } \\
\text { used as mouthrinse)/14 days }\end{array}$ & $\begin{array}{c}\text { A: Placebo } \\
\text { B: L. rhamnosus, B. } \\
\text { longum and } \\
\text { S. cereviasae } \\
\text { C: B. coagulans }\end{array}$ & $\begin{array}{l}\text { Statistically significant MS } \\
\text { reduction in groups B and C }\end{array}$ & good & - \\
\hline $\begin{array}{l}\text { Lexner et al., } \\
2010[26]\end{array}$ & $\begin{array}{l}\text { MS and Lb in } \\
\quad \text { saliva } \\
\text { (plate culturing) }\end{array}$ & $\begin{array}{l}18 \text { adolescents } \\
(13-17 \text { years })\end{array}$ & $\begin{array}{l}\text { Lactobacillus rhamnosus } \\
\text { LB21 }\left(10^{7} \mathrm{CFU} / \mathrm{mL}\right)\end{array}$ & Milk once daily/2 weeks & $\begin{array}{l}\text { A: Probiotic } \\
\text { B: Placebo }\end{array}$ & $\begin{array}{l}\text { No statistically significant } \\
\text { MS reduction and Lb }\end{array}$ & poor & 0.539 \\
\hline $\begin{array}{l}\text { Cildir et al., } \\
2009 \text { [27] } \\
\text { cross-over study }\end{array}$ & $\begin{array}{l}\text { MS and Lb in } \\
\text { saliva } \\
\text { (chair-side tests) }\end{array}$ & $\begin{array}{l}24 \text { adolescents with } \\
\text { fixed orthodontics } \\
\text { (12-16 years) }\end{array}$ & $\begin{array}{l}\text { Bifidobacterium animalis } \\
\text { subsp. lactis } \mathrm{DN} 173010 \\
\quad\left(2 \times 10^{8} \mathrm{CFU} / \mathrm{g}\right)\end{array}$ & Yogurt once daily/2 weeks & $\begin{array}{l}\text { A: Probiotic } \\
\text { B: Placebo }\end{array}$ & $\begin{array}{l}\text { Statistically significant MS } \\
\text { reduction in group A and no } \\
\text { significant Lb alterations }\end{array}$ & poor & 0.975 \\
\hline $\begin{array}{l}\text { Stecksén-Blicks } \\
\text { et al., } 2009[28]\end{array}$ & $\begin{array}{l}\text { MS and } \mathrm{Lb} \text { in } \\
\text { plaque } \\
\text { (plate culturing) }\end{array}$ & $\begin{array}{l}248 \text { children } \\
(1-4 \text { years })\end{array}$ & $\begin{array}{l}\text { Lactobacillus rhamnosus } \\
\text { LB21 }\left(10^{7} \mathrm{CFU} / \mathrm{mL}\right)\end{array}$ & Milk/21 months & $\begin{array}{l}\text { A: Probiotic/fluoride } \\
\text { B: Placebo }\end{array}$ & $\begin{array}{l}\text { No statistically significant } \\
\text { changes in MS and Lb }\end{array}$ & good & 2.462 \\
\hline $\begin{array}{c}\text { Näse et al., } \\
2001[36]\end{array}$ & $\begin{array}{l}\text { MS in plaque and } \\
\text { saliva } \\
\text { (chair-side tests) }\end{array}$ & $\begin{array}{l}594 \text { children } \\
(1-6 \text { years })\end{array}$ & $\begin{array}{l}\text { Lactobacillus rhamnosus } \\
\text { GG, ATCC } 53103 \\
\left(5-10 \times 10^{5} \mathrm{CFU} / \mathrm{mL}\right)\end{array}$ & Milk five daily/7 months & $\begin{array}{l}\text { A: Milk/probiotic } \\
\text { B: Milk }\end{array}$ & $\begin{array}{l}\text { Statistically significant MS } \\
\text { reduction in group A }\end{array}$ & excellent & 1.667 \\
\hline
\end{tabular}

Statistically significant MS decrease immediately after probiotic use in group B; recolonization during the 5 consecutive weeks. In nificant MS reduction that enhances during the 5 consecutive weeks

charomyces cereviasae Powders (dissolved in water and Billus coagulans (150 million)

tobacillus rhamnosu $\left.0^{5} \mathrm{CFU} / \mathrm{mL}\right)$
B: Milk 
Table 3. Studies with caries risk factors as outcome (adults).

\begin{tabular}{|c|c|c|c|c|c|c|c|c|}
\hline $\begin{array}{c}\text { Reference } \\
\text { Study design }\end{array}$ & Outcome(s) & Subjects Age & $\begin{array}{c}\text { Strain } \\
\text { (Concentration) }\end{array}$ & $\begin{array}{c}\text { Delivery System/ } \\
\text { Treatment } \\
\text { Duration }\end{array}$ & Groups & Results & Consort score & IF score \\
\hline $\begin{array}{l}\text { Marttinen et al., } \\
\quad 2012[18] \\
\text { Cross-over study }\end{array}$ & $\begin{array}{c}\text { Plaque } \\
\text { acidogenicity, MS } \\
\text { and Lb in plaque } \\
\text { (plate culturing) }\end{array}$ & $\begin{array}{c}13 \text { adults } \\
\text { (mean } 25 \text { years) }\end{array}$ & $\begin{array}{l}\text { Lactobacillus } \\
\text { rhamnosus } \mathrm{GG} \text { or } \\
\text { Lactobacillus reuteri } \\
\text { (196 million } \\
\text { CFU/tablet) }\end{array}$ & $\begin{array}{l}\text { Tablet twice a } \\
\text { day/2 weeks }\end{array}$ & $\begin{array}{c}\text { A: } \mathrm{LGG} \\
\text { B: L. reuteri }\end{array}$ & $\begin{array}{c}\text { No changes in plaque } \\
\text { acidogenicity. MS remained } \\
\text { stable, while Lb increased in the } \\
\text { L. reuteri group, but not } \\
\text { in the LGG group }\end{array}$ & good & 2.364 \\
\hline $\begin{array}{c}\text { Keller \& } \\
\text { Twetman, } \\
2012 \text { [19] } \\
\text { Cross-over study }\end{array}$ & $\begin{array}{c}\text { MS and } \\
\text { Lb in saliva } \\
\text { (chair-side tests) } \\
\text { Lactatic Acid } \\
\text { production in } \\
\text { plaque } \\
\text { Inhibiting }\end{array}$ & $\begin{array}{c}18 \text { adults } \\
\text { (mean } 26 \text { years) }\end{array}$ & $\begin{array}{c}\text { Lactobacillus reuteri } \\
\text { (DSM } 17938 \text { and } \\
\text { ATCC PTA 5289) } \\
\left(2 \times 10^{8} \text { CFU/tablet) }\right.\end{array}$ & $\begin{array}{l}\text { Tablets three times } \\
\text { a day/2 weeks }\end{array}$ & $\begin{array}{l}\text { A: L. reuteri } \\
\text { B: Placebo }\end{array}$ & $\begin{array}{l}\text { No statistically significant MS } \\
\text { change; Lb increased } \\
\text { significantly in group A. } \\
\text { No significant differences in } \\
\text { Lactatic Acid production }\end{array}$ & good & - \\
\hline $\begin{array}{l}\text { Keller et al., } \\
2012[20]\end{array}$ & $\begin{array}{l}\text { regrowth of } \\
\text { salivary MS after } \\
\text { full-mouth } \\
\text { disinfection } \\
\text { (chair-side tests) }\end{array}$ & $\begin{array}{c}62 \text { adults } \\
\text { (mean } 23 \text { years) }\end{array}$ & $\begin{array}{l}\text { Lactobacillus reuteri } \\
\text { (DSM } 17938 \text { and } \\
\text { ATCC PTA 5289) } \\
\left(2 \times 10^{8} \text { CFU/tablet) }\right.\end{array}$ & $\begin{array}{l}\text { Tablets twice } \\
\text { daily/6 weeks }\end{array}$ & $\begin{array}{l}\text { A: Probiotics } \\
\text { B: Placebo }\end{array}$ & $\begin{array}{l}\text { L. reuteri did not seem to affect } \\
\text { or delay the regrowth of MS }\end{array}$ & good & 2.328 \\
\hline $\begin{array}{l}\text { Petersson et al., } \\
\quad 2011[24]\end{array}$ & $\begin{array}{c}\text { MS and } \\
\text { Lb in saliva } \\
\text { (chair-side tests) } \\
\text { and plaque } \\
\text { (plate culturing) }\end{array}$ & $\begin{array}{c}160 \text { adults } \\
(58-84 \text { years })\end{array}$ & $\begin{array}{l}\text { Lactobacillus } \\
\text { rhamnosus LB21 } \\
\left(10^{7} \mathrm{CFU} / \mathrm{mL}\right)\end{array}$ & $\begin{array}{l}\text { Milk once daily/ } \\
15 \text { months }\end{array}$ & $\begin{array}{l}\text { A: Placebo } \\
\text { B: Fluoride/ } \\
\text { probiotic } \\
\text { C: Probiotic } \\
\text { D: Fluoride }\end{array}$ & $\begin{array}{c}\text { Lower prevalence of MS and Lb, } \\
\text { but not statistically significant }\end{array}$ & good & 1.066 \\
\hline $\begin{array}{c}\text { Chuang et al., } \\
2011 \text { [25] }\end{array}$ & $\begin{array}{c}\text { MS and Lb in } \\
\text { saliva (chair-side } \\
\text { tests) and } \\
\text { buffer capacity } \\
\text { (Dentobuff strip) }\end{array}$ & $\begin{array}{c}80 \text { adults } \\
\text { (20-26 years) }\end{array}$ & $\begin{array}{c}\text { Lactobacillus } \\
\text { paracasei } \\
\text { GMNL-33 } \\
\left(3 \times 10^{8} \mathrm{CFU} / \mathrm{mL}\right)\end{array}$ & $\begin{array}{c}\text { Tablets three times } \\
\text { per day/2 weeks }\end{array}$ & $\begin{array}{l}\text { A: Probiotics } \\
\text { B: Xylitol }\end{array}$ & $\begin{array}{c}\text { No statistically significant } \\
\text { differences in MS and Lb and } \\
\text { buffer capacity. MS reduction } \\
\text { intra probiotics group }\end{array}$ & poor & 2.364 \\
\hline
\end{tabular}


Table 3. Cont.

\begin{tabular}{|c|c|c|c|c|c|c|c|c|}
\hline $\begin{array}{l}\text { Caglar. et al., } \\
\text { 2008 [29] } \\
\text { Cross-over study }\end{array}$ & $\begin{array}{c}\text { MS and } \\
\text { Lb in saliva } \\
\text { (chair-side tests) }\end{array}$ & $\begin{array}{c}24 \text { adults } \\
\text { (mean } 20 \text { years) }\end{array}$ & $\begin{array}{l}\text { Bifidobacterium lactis } \\
\mathrm{Bb}-12\left(10^{7} \mathrm{CFU} / \mathrm{g}\right)\end{array}$ & $\begin{array}{l}\text { Ice-cream once } \\
\text { daily/10 days }\end{array}$ & $\begin{array}{l}\text { A: Probiotic } \\
\text { B: Placebo }\end{array}$ & $\begin{array}{l}\text { Statistically significant MS } \\
\text { reduction in group A; salivary } \\
\text { Lb levels unaltered }\end{array}$ & poor & 1.095 \\
\hline $\begin{array}{c}\text { Caglar et al., } \\
2008 \text { [30] }\end{array}$ & $\begin{array}{c}\text { MS and } \\
\text { Lb in saliva } \\
\text { (chair-side tests) }\end{array}$ & $\begin{array}{c}20 \text { women } \\
\text { (mean } 20 \text { years) }\end{array}$ & $\begin{array}{c}\text { Lactobacillus reuteri } \\
\text { ATCC 55730: ATCC } \\
\text { PTA } 528910: 1 \\
\left(1.1 \times 10^{8} \mathrm{CFU}\right)\end{array}$ & $\begin{array}{l}\text { Lozenge once } \\
\text { daily/10 days }\end{array}$ & $\begin{array}{l}\text { A: Probiotic } \\
\text { B: Placebo }\end{array}$ & $\begin{array}{l}\text { Statistically significant MS } \\
\text { reduction in group A; } \\
\text { Lb unaltered }\end{array}$ & poor & 1.072 \\
\hline $\begin{array}{c}\text { Caglar et al., } \\
2007 \text { [31] }\end{array}$ & $\begin{array}{c}\text { MS and } \\
\text { Lb in saliva } \\
\text { (chair-side tests) }\end{array}$ & $\begin{array}{c}80 \text { adults } \\
\text { (21-24 years) }\end{array}$ & $\begin{array}{l}\text { Lactobacilli reuteri } \\
\text { ATCC and } \\
\text { Lactobacilli reuteri } \\
\text { ATCC PTA } 5289 \\
\left(10^{8} \mathrm{CFU} / \mathrm{gum}\right)\end{array}$ & $\begin{array}{l}\text { chewing gums } \\
\text { three times daily/ } \\
3 \text { weeks }\end{array}$ & $\begin{array}{c}\text { A: Probiotics } \\
\text { B: Xylitol } \\
\text { C: Probiotics/xylitol } \\
\text { D: Placebo }\end{array}$ & $\begin{array}{c}\text { Statistically significant MS } \\
\text { reduction in group A, } \\
\text { B and C; Probiotic + xylitol not } \\
\text { enhance the efficacy. }\end{array}$ & poor & 1.956 \\
\hline $\begin{array}{c}\text { Caglar et al., } \\
2006[32]\end{array}$ & $\begin{array}{l}\text { MS and Lb } \\
\text { in saliva } \\
\text { (chair-side tests) }\end{array}$ & $\begin{array}{c}120 \text { adults } \\
(21-24 \text { years })\end{array}$ & $\begin{array}{l}\text { Lactobacillus reuteri } \\
\text { ATCC } 55730 \\
\left(10^{8} \mathrm{CFU} / \text { straw }\right. \\
\text { or tablet })\end{array}$ & $\begin{array}{c}\text { Water or tablet } \\
\text { once daily/3 weeks }\end{array}$ & $\begin{array}{l}\text { A: Water/probiotic } \\
\text { B: Placebo water } \\
\text { C: Tablet/probiotic } \\
\text { D: Placebo tablet }\end{array}$ & $\begin{array}{l}\text { Statistically significant MS } \\
\text { reduction in groups A and C; } \\
\text { similar but non-significant } \\
\text { trend for Lb }\end{array}$ & poor & 1.017 \\
\hline $\begin{array}{l}\text { Caglar et al., } \\
2005[33] \\
\text { Cross-over study }\end{array}$ & $\begin{array}{c}\text { MS and } \\
\text { Lb in saliva } \\
\text { (chair-side tests) }\end{array}$ & $\begin{array}{c}26 \text { adults } \\
\text { (21-24 years) }\end{array}$ & $\begin{array}{c}\text { Bifidobacterium } \\
\text { DN-173010 } \\
\left(7 \times 10^{7} \mathrm{CFU} / \mathrm{g}\right)\end{array}$ & $\begin{array}{l}\text { Yogurt once } \\
\text { daily/2 weeks }\end{array}$ & $\begin{array}{l}\text { A: Probiotic } \\
\text { B: Placebo }\end{array}$ & $\begin{array}{c}\text { Statistically significant MS } \\
\text { reduction in group A; similar but } \\
\text { non-significant trend for } \mathrm{Lb}\end{array}$ & poor & 0.783 \\
\hline $\begin{array}{c}\text { Montalto et al., } \\
2004 \text { [34] }\end{array}$ & $\begin{array}{c}\text { MS and } \\
\text { Lb in saliva } \\
\text { (chair-side tests) }\end{array}$ & $\begin{array}{c}35 \text { adults } \\
\text { (23-37 years) }\end{array}$ & $\begin{array}{l}\text { L. sporogens, } \\
\text { L. bifidum, } \\
\text { L. bulgaricus, } \\
\text { L. termophilus, } \\
\text { L. acidophilus, } \\
\text { L. casei, } \\
\text { L. rhamnosus } \\
\text { (1.88 } \times 10^{9} \text { live } \\
\text { cells/day) }\end{array}$ & $\begin{array}{c}\text { Liquid and } \\
\text { capsule/45 days }\end{array}$ & $\begin{array}{c}\text { A: Probiotics } \\
\text { capsules placebo in } \\
\text { liquid } \\
\text { B: Liquid probiotics } \\
\text { placebo in capsules } \\
\text { C: Placebo in both } \\
\text { liquid and capsule }\end{array}$ & $\begin{array}{c}\text { Statistically significant Lb } \\
\text { increase in groups A and B. } \\
\text { MS not significantly modified. }\end{array}$ & poor & 1.473 \\
\hline
\end{tabular}


Table 3. Cont.

\begin{tabular}{|c|c|c|c|c|c|c|c|c|}
\hline $\begin{array}{l}\text { Ahola et al., } \\
2002[35]\end{array}$ & $\begin{array}{c}\text { MS, Lb and } \\
\text { yeasts in saliva } \\
\text { (chair-side tests) } \\
\text { and buffer } \\
\text { capacity } \\
\text { (Dentobuff strip) }\end{array}$ & $\begin{array}{l}74 \text { young adults } \\
\text { (18-35 years) }\end{array}$ & $\begin{array}{c}\text { Lactobacillus } \\
\text { rhamnosus GG ATCC } \\
53103\left(1.9 \times 10^{7}\right. \\
\text { CFU/g) and } \\
\text { Lactobacillus } \\
\text { rhamnosus } \text { LC } 705 \\
\left(1.2 \times 10^{7} \mathrm{CFU} / \mathrm{g}\right)\end{array}$ & $\begin{array}{l}\text { Cheese five daily/ } \\
3 \text { weeks }\end{array}$ & $\begin{array}{l}\text { A: Probiotics } \\
\text { B: Placebo }\end{array}$ & $\begin{array}{l}\text { No statistically significant } \\
\text { differences in MS and Lb after } \\
\text { the intervention; during } \\
\text { the post-treatment period } \\
\text { ( } 3 \text { weeks) a significantly } \\
\text { reduction of the two species in } \\
\text { group A. No statistically } \\
\text { significant differences in yeast } \\
\text { and buffer capacity }\end{array}$ & good & 1.047 \\
\hline Reference & Outcome(s) & Subjects & $\begin{array}{c}\text { Strain } \\
\text { (Concentration) }\end{array}$ & $\begin{array}{c}\text { Delivery System/ } \\
\text { Treatment Duration }\end{array}$ & Groups & Results & Consort score & IF score \\
\hline $\begin{array}{c}\text { Taipale et al., } \\
2013[14]\end{array}$ & $\begin{array}{l}\text { Caries } \\
\text { increment } \\
\text { (ICDAS } \\
\text { index) }\end{array}$ & $\begin{array}{l}106 \text { children } \\
(4 \text { years })\end{array}$ & $\begin{array}{l}\text { Bifidobacterium } \\
\text { animalis subsp. } \\
\text { lactis } \mathrm{BB}-12 \\
(1010 \mathrm{CFU} / \mathrm{mL})\end{array}$ & $\begin{array}{c}\text { Tablets in } \\
\text { slow-release pacifier or } \\
\text { spoon twice daily/ } \\
22-23 \text { months }\end{array}$ & $\begin{array}{l}\text { A: Probiotic } \\
\text { B: Xylitol } \\
\text { C: Sorbitol }\end{array}$ & $\begin{array}{c}\text { No differences in the } \\
\text { occurrence } \\
\text { of enamel caries }\end{array}$ & excellent & 2.328 \\
\hline $\begin{array}{l}\text { Petersson et al., } \\
2011 \text { [24] }\end{array}$ & $\begin{array}{c}\text { Root Caries } \\
\text { Index (RCI) } \\
\text { and Electric } \\
\text { Resistance } \\
\text { Measurements } \\
\text { (ERM) }\end{array}$ & $\begin{array}{c}160 \text { adults } \\
\text { (58-84 years) }\end{array}$ & $\begin{array}{c}\text { Lactobacillus } \\
\text { rhamnosus } \mathrm{LB} 21\left(10^{7}\right. \\
\mathrm{CFU} / \mathrm{mL})\end{array}$ & $\begin{array}{l}\text { Milk once daily/ } \\
15 \text { months }\end{array}$ & $\begin{array}{c}\text { A: Placebo } \\
\text { B: } \\
\text { Fluoride/probiotic } \\
\text { C: Probiotic } \\
\text { D: Fluoride }\end{array}$ & $\begin{array}{l}\text { Higher numbers of RCI } \\
\text { reversals in groups B, C and } \\
\text { D. Mean ECM values } \\
\text { increased significantly in } \\
\text { groups A, B and C }\end{array}$ & good & 1.066 \\
\hline $\begin{array}{l}\text { Stecksén-Blicks } \\
\text { et al., } 2009 \text { [28] }\end{array}$ & $\begin{array}{c}\text { Caries } \\
\text { increment } \\
(\mathrm{dmfs} \text { index })\end{array}$ & $\begin{array}{l}248 \text { children } \\
(1-4 \text { years })\end{array}$ & $\begin{array}{l}\text { Lactobacillus } \\
\text { rhamnosus } \mathrm{LB} 21 \\
\left(10^{7} \mathrm{CFU} / \mathrm{mL}\right)\end{array}$ & $\begin{array}{c}\text { Milk once } \\
\text { daily/21 months }\end{array}$ & $\begin{array}{c}\text { A: Probiotic/ } \\
\text { fluoride } \\
\text { B: Placebo }\end{array}$ & $\begin{array}{c}\text { Statistically significant } \\
\text { difference in caries increment } \\
\text { in group A }\end{array}$ & good & 2.462 \\
\hline
\end{tabular}




\subsection{Probiotics and Caries Prevention in Children/Adolescents}

Eleven studies were evaluated [14-17,21-23,26-28,36]. Only one study was performed to verify the effect of the early administration of probiotics (Bifidobacterium animalis subsp. lactis BB-12) on the oral colonization of mutans streptococci (MS) in 106 infants from a low-caries population [17]. Subjects received probiotic bacteria, xylitol or sorbitol (polyol 100-300 mg) from the age of 1-2 months to the age of 2 years, twice a day. The MS concentration in plaque of the mothers at the start of the study was high and similar in all subjects, without significant differences. At the end of the study, children showed a rather low MS colonization percentage, with a statistically significant difference among groups. At the age of 4 years, the same children were re-evaluated to assess the MS level in plaque and the occurrence of dental caries in deciduous teeth [14]. No differences were observed for both parameters among the three groups.

Otherwise, nine studies were carried out to verify the effect of probiotics strains on MS levels in saliva and/or dental plaque, using different vehicle [15,16,21-23,26-28,36]. Only two studies did not demonstrate any change in SM level [26,28].

The effect of milk containing L. rhamnosus on MS counts was evaluated in four papers (two short and two long-term studies). In the short-term studies [16,26], the effect of milk containing Lactobacillus rhamnosus (hct 70 or LB21) for few weeks was registered in small groups of adolescents. The difference in post treatment regarding MS count between test and control group was not statistically significant, while the difference in follow-up was highly significant [16]. No statistically significant differences in SM were recorded in subjects who received milk with probiotic compared to subjects using milk without probiotic [26]. In the long-term studies [28,36], L. rhamnosus was administered for several months (7 and 21 months respectively). Statistically significant reductions were recorded with Lactobacillus rhamnosus GG, ATCC use [36], while no statistically significant changes were observed in SM counts in subjects receiving Lactobacillus rhamnosus LB21 [28].

Two studies were performed with yogurt as probiotics vehicle [22,27]. The effect of the administration of yogurt containing Lactobacillus rhamnosus GG for three weeks in 105 children was evaluated with a significant decrease in SM count immediately after probiotics use alone, but recolonization was described during the five consecutive weeks [22]. Pre-treatment with chlorhexidine produced a statistically significant reduction in salivary SM counts that enhances during the five consecutive weeks. A double-blind, crossover study was carried out on 24 healthy adolescents, undergoing orthodontic treatment, with the aim to assess the effect of yogurt containing Bifidobacterium animalis subsp. lactis DN-173010 administered once daily [27]. Statistically significant reduction of MS was recorded after probiotic yogurt consumption.

One study used ice-cream as probiotic vehicle [21]; a combination of Bifidobacterium lactis Bb-12 and Lactobacillus acidophilus La-5 was evaluated in 40 adolescents. Significant reduction in salivary MS scores was reported after consumption of the probiotic compared to baseline.

One study was performed using lozenges as probiotic vehicle. The effect of lozenges containing Lactobacillus brevis CD2 administered for six weeks was evaluated in 191 high caries risk children [15]. A statistically significant reduction of the cariogenic microorganism was recorded.

One study used two powders as probiotic vehicle in 150 children aged 7-14 years, containing the first Lactobacillus rhamnosus, Bifidobacterium longum and Saccharomyces cereviasae and the second 
Bacillus coagulans, and compared them to a placebo powder [23]. Powders dissolved in $20 \mathrm{~mL}$ of water were used as a mouth rinse for one minute for 14 consecutive days. Data analysis showed a statistically significant reduction in MS counts in both probiotics groups.

Five studies of the ten reported above, investigated the effect of the probiotics strain on Lb level also [17,21,26-28]. In all studies, a statistically significant change in Lb counts in saliva and/or plaque was not observed. Moreover, one study evaluated the effect of the probiotic on oral yeasts, failing to prove any statistically effect [17]. The effect of probiotics on plaque $\mathrm{pH}$ modification after a rinse with a $10 \%$ sugared solution was investigated and plaque acidogenicity resulted significantly lower in subjects that have used probiotic lozenges [15]. Two studies evaluated the probiotic effect on caries lesion development [14,28]. A statistically significant difference in caries increment was recorded only in one paper in subjects who received probiotic and fluoride compared to subjects who received placebo milk [28].

\subsection{Probiotics and Caries Prevention in Adults}

Twelve studies were selected [18-20,24,25,29-35]: all of them investigated the effects of probiotic administration on MS counts in plaque and/or saliva and six demonstrated a MS reduction.

Caglar and co-workers [29-33], performed several studies on the change of salivary MS concentration after the use of several probiotics (Bifidobacterium lactis $\mathrm{Bb}-12$, Lactobacillus reuteri ATCC 55730 and ATCC PTA 5289, Bifidobacterium DN-173 010) using different vehicles (ice-cream, chewing-gum, water, yogurt and tablets). MS concentrations decrease significantly in all studies.

No statistically significant differences in MS counts were recorded immediately after consumption of cheese containing Lactobacillus rhamnosus GG and Lactobacillus rhamnosus LC 705, but a significant reduction was reported three weeks after the experimental period [35].

Conversely, the other six of the twelve studies did not reveal an effect of probiotics administration on MS counts [18-20,24,25,34]. Four short-term studies were performed using tablets containing Lactobacillus rhamnosus or Lactobacllus reuteri; MS counts remained stable after the administration of both probiotics twice a day for two weeks in 13 adults [18]. No significant differences were also observed after the use for two weeks of Lactobacillus reuteri on MS counts in 18 adults [19], and using the same strains after full mouth disinfection with chlorhexidine on 62 adults on regrow of MS [20]. Tablets containing Lactobacillus paracasei GMNL-33 were unsuccessfully administered to 80 young adults [25]. One long-term study evaluated the effect of Lactobacillus rhamnosus LB21 delivered in milk on MS count in saliva and supra-gingival plaque in 160 older adults for 15 months [24]. No statistically significant reduction in MS count was registered. Results from a study utilizing several strains of Lactobacillus spp. in liquid and capsules form in 35 adults revealed no significantly MS count reduction [34].

Moreover, ten studies of the twelve reported above, investigated the effect of the probiotics strain on Lb level in saliva and/or plaque [18,19,25,29,30-35]. Eight studies failed to prove any effect on $\mathrm{Lb}$ counts and two studies demonstrated a statistically significant change in Lb counts $[19,35]$.

Two studies evaluated also the effect of probiotics on plaque acidogenicity, but no significant changes were found $[18,19]$. Two studies investigated the effect on buffer capacity failing to demonstrate 
a statistically significant difference on it $[25,35]$. One of these did not demonstrate an effect on oral yeast yet [35].

\section{Discussion}

The role of the administration of probiotic strains in caries prevention was the aim of this systematic review. Results described by various research groups were encouraging [15-17,21-24,27,29-33,35,36], but the scientific evidence is still unclear and often not very high. The main goal for the use of probiotics in caries prevention is to replace and displace cariogenic bacteria, mainly mutans streptococci, with noncariogenic bacteria [13].

Most clinical trials reviewed had a small sample size and reported caries risk factors as intermediate or surrogate endpoints, which limited the conclusions about the real efficacy of probiotics administration in caries lesion prevention. From the analysis of the RCTs selected, it reasonable to affirm that probiotic strains may play a role as antagonistic agent on cariogenic bacteria. In the two-thirds of the selected papers, probiotics have demonstrated the capacity to reduce MS counts in saliva and/or plaque regardless of the product or strain used. However, this effect is variable and probably short-lasting. In addition, MS are no longer considered the main cariogenic bacteria involved in the caries progress, since the important role of non-mutans acidogenic and aciduric bacteria was clarified [5]. Different results are reported on the effect of probiotics on lactobacilli counts. From the fourteen studies that evaluated the changes of this interim outcome, just two reported a positive result $[19,35]$. The other interim outcomes considered (yeasts and plaque acidogenicity) were investigated in few studies and the results are unclear. Only three selected papers [14,24,28], two performed on children and another one on adults/elderly samples, had caries lesion development as outcome; two studies reported a statistically significant difference in caries increment after 15/21 months of probiotics use [24,28].

Several mechanisms of action for probiotic are described in literature, same of them still not fully understood. Several local and systemic effects are describing, including adhesion, co-aggregation, competitive inhibition, production of organic acids and bacteriocin-like compounds and immune-modulation [86]. However, probiotic bacteria are not able to colonize oral cavity permanently [29], so a continuous regular, almost daily intake is required. This may be a compliance aspect to be considered.

In eleven selected papers, a dairy product (milk, cheese, yogurt and ice-cream) was used as delivery vehicle for probiotics [16,21,22,24,26-29,35,36]. These non-sweetened products are known to possess caries preventive effects related to a natural high contents in calcium and phosphate that enhance remineralization of hard oral tissues and contrast acids produced by cariogenic bacteria after sugared foods and drinks intake [13]. Only one selected paper used chewing gums as delivery vehicle [31]. The use of non-sugared chewing gum has been considered useful for dental health, since it reduces plaque acidogenicity and increases enamel remineralization, enhancing salivary flow rate [87]. The remaining eleven papers used as probiotic vehicle products (lozenges, tablets, powders) without any reported preventive effects themselves [14,15,17-20,23,25,30,32,34].

One study evaluated the combined effect of probiotics and fluoride on cariogenic bacteria and caries lesion increment. No statistically significant differences were recorded between the group using probiotics alone and those using probiotic and fluoride together [24]. Another paper studied the 
combined effect of probiotics and low dosage of xylitol on cariogenic microorganisms [31], but no statistically significant differences were noted compared to probiotics alone. Finally only one study has investigated the effect of probiotic on MS counts after chlorhexidine mouthwash disinfection [22]. Pre-treatment with chlorhexidine produced a long-lasting reduction in salivary SM compared to probiotics alone.

It is interesting to note that up to day none products have successfully approved by the European Food Safety Authority (EFSA) [88,89].

A theoretical risk of the probiotic assumption is the increase of caries risk due to the capacity of probiotic strains to form biofilm and produce acids, but this aspect was not taken into consideration by any papers.

Two approaches have been used to assess the quality of RCTs in the present review: the CONSORT checklist and the journal Impact Factor. A significant association between the CONSORT score and the impact factor was reported [90].

The CONSORT checklist takes into account 25 important methodological items, providing an accurate evaluation of the methodological correctness with which the study was planned and carried out. From the analysis of the checklists of the selected papers, the main deficiencies observed were the lack of information on methods to define the hypothesis, the sample size calculation, the absence of data on the results of estimated effects size and their precision. These methodological weaknesses reduce the validity of studies and the interpretation of the results may lead to biased findings. Moreover, few studies reported correctly the results of the RCTs not taking into account other sources of bias. In general, the quality of reporting of RCTs was quite low, with half of all studies scoring as poor with the exception of three studies that were scored as excellent. These results are similar to those reported of other systematic review [13], but it is possible to observe a progressive improvement in the scientific evidence of the effect of probiotic on caries prevention.

The journal impact factor has been used widely as a quality measure of the published papers [91]. All selected studies except two were published in journals with impact factor and all except one in dental journal [34]. The mean value of impact factors of the selected studies (1.438) might seem low when compared with IF of journals from other areas of medicine. However, the mean impact factor value of the 81 impacted dental journals is quite low (1.455-range 0.037-3.961), with the journals with the highest IF values dedicated to other topic of dentistry, different from caries prevention. Therefore, the mean IF value recorded in the present review has to be considered a quite good score.

\section{Conclusions}

The use of probiotic strains for caries prevention showed promising results even if only few studies have demonstrated clear clinical outcomes. Therefore, the scientific evidence is still poor. A continuous regular almost daily intake is probably required; this maybe a compliance aspect to be considered. However, for all products effective in caries prevention (i.e., fluoride and chlorhexidine) a frequent intake is required, so a possible way of administration could be to insert probiotic in other daily preventive products like toothpaste. 


\section{Conflict of Interest}

The authors declare no conflict of interest. The authors declare that no support was supplied by probiotic companies.

\section{References}

1. Campus, G.; Solinas, G.; Cagetti, M.G.; Senna, A.; Minelli, L.; Majori, S.; Montagna, M.T.; Reali, D.; Castiglia, P.; Strohmenger, L. National pathfinder survey of 12-year-old children's oral health in Italy. Caries Res. 2007, 41, 512-517.

2. Campus, G.; Solinas, G.; Strohmenger, L.; Cagetti, M.G.; Senna, A.; Minelli, L.; Majori, S.; Montagna, M.T.; Reali, D.; Castiglia, P. Collaborating Study Group. National pathfinder survey on children's oral health in Italy: Pattern and severity of caries disease in 4-year-olds. Caries Res. 2009, 43, 155-162.

3. Marja-Leena, M.; Paivi, R.; Sirkka, J.; Ansa, O.; Matti, S. Childhood caries is still in force: A 15-year follow-up. Acta Odontol. Scand. 2008, 66, 189-192.

4. Selwitz, R.H.; Ismail, A.I.; Pitts, N.B. Dental caries. Lancet 2007, 369, 51-59.

5. Takahashi, N.; Nyvad, B. The role of bacteria in the caries process: Ecological perspectives. J. Dent. Res. 2011, 90, 294-303.

6. Eliasson, L.; Carlén, A.; Almståhl, A.; Wikström, M.; Lingström, P. Dental plaque pH and micro-organisms during hyposalivation. J. Dent. Res. 2006, 85, 334-338.

7. Milgrom, P.; Söderling, E.M.; Nelson, S.; Chi, D.L.; Nakai, Y. Clinical evidence for polyol efficacy. Adv. Dent. Res. 2012, 24, 112-116.

8. Petersen, P.E.; Lennon, M.A. Effective use of fluorides for the prevention of dental caries in the 21st century: The WHO approach. Community Dent. Oral Epidemiol. 2004, 32, 319-321.

9. Zero, D.T. Dentifrices, mouthwashes and remineralization/caries arrestment strategies. BMC Oral Health 2006, 6 (Suppl. 1), 9.

10. World Health Organization. Available online: http://www.who.int/foodsafety/publications/ fs_management/en/probiotics.pdf(accessed on 17 April 2013).

11. Floch, M.H.; Walker, W.A.; Madsen, K.; Sanders, M.E.; Macfarlane, G.T.; Flint, H.J.; Dieleman, L.A.; Ringel, Y.; Guandalini, S.; Kelly, C.P.; et al. Recommendations for probiotic use-2011 update. J. Clin. Gastroenterol. 2011, 4, S168-S171.

12. Saha, S.; Tomaro-Duchesneau, C.; Tabrizian, M.; Prakash, S. Probiotics as oral health biotherapeutics. Expert Opin. Biol. Ther. 2012, 12, 1207-1220.

13. Twetman, S.; Keller, M.K. Probiotics for caries prevention and control. Adv. Dent. Res. 2012, 24, 98-102.

14. Taipale, T.; Pienihäkkinen, K.; Alanen, P.; Jokela, J.; Söderling, E. Administration of Bifidobacterium animalis subsp. lactis BB-12 in early childhood: A post-trial effect on caries occurrence at four years of age. Caries Res. 2013, 47, 364-372.

15. Campus, G.; Cocco, F.; Carta, G.; Cagetti, M.G.; Simark-Mattson, C.; Strohmenger, L.; Lingström, P. Effect of a daily dose of Lactobacillus brevis CD2 lozenges in high caries risk schoolchildren. Clin. Oral Investig. 2013, doi:10.100.7/s00784-013-09-80-9. 
16. Juneja, A.; Kakade, A. Evaluating the effect of probiotic containing milk on salivary mutans streptococci levels. J. Clin. Pediatr. Dent. 2012, 37, 9-14.

17. Taipale, T.; Pienihäkkinen, K.; Salminen, S.; Jokela, J.; Söderling, E. Bifidobacterium animalis subsp. lactis BB-12 administration in early childhood: A randomized clinical trial of effects on oral colonization by mutans streptococci and the probiotic. Caries Res. 2012, 46, 69-77.

18. Marttinen, A.; Haukioja, A.; Karjalainen, S.; Nylund, L.; Satokari, R.; Öhman, C.; Holgerson, P.; Twetman, S.; Söderling, E. Short-Term consumption of probiotic lactobacilli has no effect on acid production of supragingival plaque. Clin. Oral Investig. 2012, 16, 797-803.

19. Keller, M.K.; Twetman, S. Acid production in dental plaque after exposure to probiotic bacteria. BMC Oral Health 2012, 12, 44.

20. Keller, M.K.; Hasslöf, P.; Dahlén, G.; Stecksén-Blicks, C.; Twetman, S. Probiotic supplements (Lactobacillus reuteri DSM 17938 and ATCC PTA 5289) do not affect regrowth of mutans streptococci after full-mouth disinfection with chlorhexidine: A randomized controlled multicenter trial. Caries Res. 2012, 46, 140-146.

21. Singh, R.P.; Damle, S.G.; Chawla, A. Salivary mutans streptococci and lactobacilli modulations in young children on consumption of probiotic ice-cream containing Bifidobacterium lactis Bb12 and Lactobacillus acidophilus La5. Acta Odontol. Scand. 2011, 69, 389-394.

22. Aminabadi, N.A.; Erfanparast, L.; Ebrahimi, A.; Oskouei, S.G. Effect of chlorhexidine pretreatment on the stability of salivary lactobacilli probiotic in six- to twelve-year-old children: A randomized controlled trial. Caries Res. 2011, 45, 148-154.

23. Jindal, G.; Pandey, R.K.; Agarwal, J.; Singh, M. A comparative evaluation of probiotics on salivary mutans streptococci counts in Indian children. Eur. Arch. Paediatr. Dent. 2011, 12, 211-215.

24. Petersson, L.G.; Magnusson, K.; Hakestam, U.; Baigi, A.; Twetman, S. Reversal of primary root caries lesions after daily intake of milk supplemented with fluoride and probiotic lactobacilli in older adults. Acta Odontol. Scand. 2011, 69, 321-327.

25. Chuang, L.C.; Huang, C.S.; Ou-Yang, L.W.; Lin, S.Y. Probiotic Lactobacillus paracasei effect on cariogenic bacterial flora. Clin. Oral Investig. 2011, 15, 471-476.

26. Lexner, M.O.; Blomqvist, S.; Dahlén, G.; Twetman, S. Microbiological profiles in saliva and supragingival plaque from caries-active adolescents before and after a short-term daily intake of milk supplemented with probiotic bacteria-A pilot study. Oral Health Prev. Dent. 2010, 8, 383-388.

27. Cildir, S.K.; Germec, D.; Sandalli, N.; Ozdemir, F.I.; Arun, T.; Twetman, S.; Caglar, E. Reduction of salivary mutans streptococci in orthodontic patients during daily consumption of yoghurt containing probiotic bacteria. Eur. J. Orthod. 2009, 31, 407-411.

28. Stecksén-Blicks, C.; Sjöström, I.; Twetman, S. Effect of long-term consumption of milk supplemented with probiotic lactobacilli and fluoride on dental caries and general health in preschool children: A cluster-randomized study. Caries Res. 2009, 43, 374-381.

29. Caglar, E.; Kuscu, O.O.; Selvi Kuvvetli, S.; Kavaloglu Cildir, S.; Sandalli, N.; Twetman, S. Short-term effect of ice-cream containing Bifidobacterium lactis Bb-12 on the number of salivary mutans streptococci and lactobacilli. Acta Odontol. Scand. 2008, 66, 154-158. 
30. Caglar, E.; Kuscu, O.O.; Cildir, S.K.; Kuvvetli, S.S.; Sandalli, N. A probiotic lozenge administered medical device and its effect on salivary mutans streptococci and lactobacilli. Int. J. Paediatr. Dent. 2008, 18, 35-39.

31. Caglar, E.; Kavaloglu, S.C.; Kuscu, O.O.; Sandalli, N.; Holgerson, P.L.; Twetman, S. Effect of chewing gums containing xylitol or probiotic bacteria on salivary mutans streptococci and lactobacilli. Clin. Oral Investig. 2007, 11, 425-429.

32. Caglar, E.; Cildir, S.K.; Ergeneli, S.; Sandalli, N.; Twetman, S. Salivary mutans streptococci and lactobacilli levels after ingestion of the probiotic bacterium Lactobacillus reuteri ATCC 55730 by straws or tablets. Acta Odontol. Scand. 2006, 64, 314-318.

33. Caglar, E.; Sandalli, N.; Twetman, S.; Kavaloglu, S.; Ergeneli, S.; Selvi, S. Effect of yogurt with Bifidobacterium DN-173 010 on salivary mutans streptococci and lactobacilli in young adults. Acta Odontol. Scand. 2005, 63, 317-320.

34. Montalto, M.; Vastola, M.; Marigo, L.; Covino, M.; Graziosetto, R.; Curigliano, V.; Santoro, L.; Cuoco, L.; Manna, R.; Gasbarrini, G. Probiotic treatment increases salivary counts of lactobacilli: A double-blind, randomized, controlled study. Digestion 2004, 69, 53-56.

35. Ahola, A.J.; Yli-Knuuttila, H.; Suomalainen, T.; Poussa, T.; Ahlström, A.; Meurman, J.H.; Korpela, R. Short-term consumption of probiotic-containing cheese and its effect on dental caries risk factors. Arch. Oral Biol. 2002, 47, 799-804.

36. Näse, L.; Hatakka, K.; Savilahti, E.; Saxelin, M.; Pönkä, A.; Poussa, T.; Korpela, R.; Meurman, J.H. Effect of long-term consumption of a probiotic bacterium, Lactobacillus rhamnosus GG, in milk on dental caries and caries risk in children. Caries Res. 2001, 35, 412-420.

37. Rao, Y.; Lingamneni, B.; Reddy, D. Probiotics in oral health-A review. J. N. J. Dent. Assoc. 2012, 83, 28-32.

38. Ravn, I.; Dige, I.; Meyer, R.L.; Nyvad, B. Colonization of the oral cavity by probiotic bacteria. Caries Res. 2012, 46, 107-112.

39. National Library of Medicine. Available online: http://www.nlm.nih.gov/mesh/MBrowser.html.22 (accessed on 14 February 2013).

40. Keller, M.K.; Bardow, A.; Jensdottir, T.; Lykkeaa, J.; Twetman, S. Effect of chewing gums containing the probiotic bacterium Lactobacillus reuteri on oral malodour. Acta Odontol. Scand. 2012, 70, 246-250.

41. Wang, S.; Zhu, H.; Lu, C.; Kang, Z.; Luo, Y.; Feng, L.; Lu, X. Fermented milk supplemented with probiotics and prebiotics can effectively alter the intestinal microbiota and immunity of host animals. J. Dairy Sci. 2012, 95, 4813-4822.

42. Allen, S.J.; Wareham, K.; Bradley, C.; Harris, W.; Dhar, A.; Brown, H.; Foden, A.; Cheung, W.Y.; Gravenor, M.B.; Plummer, S.; et al. A multicentre randomised controlled trial evaluating lactobacilli and bifidobacteria in the prevention of antibiotic-associated diarrhoea in older people admitted to hospital: The PLACIDE study protocol. BMC Infect. Dis. 2012, 12, 108.

43. Iniesta, M.; Herrera, D.; Montero, E.; Zurbriggen, M.; Matos, A.R.; Marín, M.J.; Sánchez-Beltrán, M.C.; Llama-Palacio, A.; Sanz, M. Probiotic effects of orally administered Lactobacillus reuteri-containing tablets on the subgingival and salivary microbiota in patients with gingivitis. A randomized clinical trial. J. Clin. Periodontol. 2012, 39, 736-744. 
44. Slawik, S.; Staufenbiel, I.; Schilke, R.; Nicksch, S.; Weinspach, K.; Stiesch, M.; Eberhard, J. Probiotics affect the clinical inflammatory parameters of experimental gingivitis in humans. Eur. J. Clin. Nutr. 2011, 65, 857-863.

45. Vandenplas, Y.; de Hert, S.G. Randomised clinical trial: The synbiotic food supplement Probiotical vs. placebo for acute gastroenteritis in children. Aliment. Pharmacol. Ther. 2011, 34, 862-867.

46. Burton, J.P.; Cowley, S.; Simon, R.R.; McKinney, J.; Wescombe, P.A.; Tagg, J.R. Evaluation of safety and human tolerance of the oral probiotic Streptococcus salivarius K12: A randomized, placebo-controlled, double-blind study. Food Chem. Toxicol. 2011, 49, 2356-2364.

47. Krauss-Silva, L.; Moreira, M.E.; Alves, M.B.; Braga, A.; Camacho, K.G.; Batista, M.R.; Almada-Horta, A.; Rebello, M.R.; Guerra, F. A randomised controlled trial of probiotics for the prevention of spontaneous preterm delivery associated with bacterial vaginosis: Preliminary results. Trials 2011, 12, 239.

48. Hummelen, R.; Changalucha, J.; Butamanya, N.L.; Koyama, T.E.; Cook, A.; Habbema, J.D.; Reid, G. Effect of 25 weeks probiotic supplementation on immune function of HIV patients. Gut Microbes 2011, 2, 80-85.

49. Harini, P.M.; Anegundi, R.T. Efficacy of a probiotic and chlorhexidine mouth rinses: A short-term clinical study. J. Indian Soc. Pedod. Prev. Dent. 2010, 28, 179-182.

50. Saxelin, M.; Lassig, A.; Karjalainen, H.; Tynkkynen, S.; Surakka, A.; Vapaatalo, H.; Järvenpää, S.; Korpela, R.; Mutanen, M.; Hatakka, K. Persistence of probiotic strains in the gastrointestinal tract when administered as capsules, yoghurt, or cheese. Int. J. Food Microbiol. 2010, 144, 293-300.

51. Hummelen, R.; Changalucha, J.; Butamanya, N.L.; Cook, A.; Habbema, J.D.; Reid, G. Lactobacillus rhamnosus GR-1 and L. reuteri RC-14 to prevent or cure bacterial vaginosis among women with HIV. Int. J. Gynaecol. Obstet. 2010, 111, 245-248.

52. Arroyo, R.; Martín, V.; Maldonado, A.; Jiménez, E.; Fernández, L.; Rodríguez, J.M. Treatment of infectious mastitis during lactation: Antibiotics versus oral administration of Lactobacilli isolated from breast milk. Clin. Infect. Dis. 2010, 50, 1551-1558.

53. Grossi, E.; Buresta, R.; Abbiati, R.; Cerutti, R. Clinical trial on the efficacy of a new symbiotic formulation, Flortec, in patients with acute diarrhea: A multicenter, randomized study in primary care. J. Clin. Gastroenterol. 2010, 44, S35-S41.

54. Sierra, S.; Lara-Villoslada, F.; Sempere, L.; Olivares, M.; Boza, J.; Xaus, J. Intestinal and immunological effects of daily oral administration of Lactobacillus salivarius CECT5713 to healthy adults. Anaerobe 2010, 16, 195-200.

55. Sinkiewicz, G.; Cronholm, S.; Ljunggren, L.; Dahlén, G.; Bratthall, G. Influence of dietary supplementation with Lactobacillus reuteri on the oral flora of healthy subjects. Swed. Dent. J. 2010, 34, 197-206.

56. Mayanagi, G.; Kimura, M.; Nakaya, S.; Hirata, H.; Sakamoto, M.; Benno, Y.; Shimauchi, H. Probiotic effects of orally administered Lactobacillus salivarius WB21-containing tablets on periodontopathic bacteria: A double-blinded, placebo-controlled, randomized clinical trial. J. Clin. Periodontol. 2009, 36, 506-513. 
57. Dommels, Y.E.; Kemperman, R.A.; Zebregs, Y.E.; Draaisma, R.B.; Jol, A.; Wolvers, D.A.; Vaughan, E.E.; Albers, R. Survival of Lactobacillus reuteri DSM 17938 and Lactobacillus rhamnosus GG in the human gastrointestinal tract with daily consumption of a low-fat probiotic spread. Appl. Environ. Microbiol. 2009, 75, 6198-6204.

58. Ranganathan, N.; Friedman, E.A.; Tam, P.; Rao, V.; Ranganathan, P.; Dheer, R. Probiotic dietary supplementation in patients with stage 3 and 4 chronic kidney disease: A 6-month pilot scale trial in Canada. Curr. Med. Res. Opin. 2009, 25, 1919-1930.

59. Twetman, S.; Derawi, B.; Keller, M.; Ekstrand, K.; Yucel-Lindberg, T.; Stecksen-Blicks, C. Short-term effect of chewing gums containing probiotic Lactobacillus reuteri on the levels of inflammatory mediators in gingival crevicular fluid. Acta Odontol. Scand. 2009, 67, 19-24.

60. Basu, S.; Paul, D.K.; Ganguly, S.; Chatterjee, M.; Chandra, P.K. Efficacy of high-dose Lactobacillus rhamnosus GG in controlling acute watery diarrhea in Indian children: A randomized controlled trial. J. Clin. Gastroenterol. 2009, 43, 208-213.

61. Staab, B.; Eick, S.; Knöfler, G.; Jentsch, H. The influence of a probiotic milk drink on the development of gingivitis: A pilot study. J. Clin. Periodontol. 2009, 36, 850-856.

62. Mao, M.; Yu, T.; Xiong, Y.; Wang, Z.; Liu, H.; Gotteland, M.; Brunser, O. Effect of a lactose-free milk formula supplemented with bifidobacteria and streptococci on the recovery from acute diarrhoea. Asia Pac. J. Clin. Nutr. 2008, 17, 30-34.

63. Shimauchi, H.; Mayanagi, G.; Nakaya, S.; Minamibuchi, M.; Ito, Y.; Yamaki, K.; Hirata, H. Improvement of periodontal condition by probiotics with Lactobacillus salivarius WB21: A randomized, double-blind, placebo-controlled study. J. Clin. Periodontol. 2008, 35, 897-905.

64. Marcone, V.; Calzolari, E.; Bertini, M. Effectiveness of vaginal administration of Lactobacillus rhamnosus following conventional metronidazole therapy: How to lower the rate of bacterial vaginosis recurrences. New Microbiol. 2008, 31, 429-433.

65. Panigrahi, P.; Parida, S.; Pradhan, L.; Mohapatra, S.S.; Misra, P.R.; Johnson, J.A.; Chaudhry, R.; Taylor, S.; Hansen, N.I.; Gewolb, I.H. Long-term colonization of a Lactobacillus plantarum synbiotic preparation in the neonatal gut. J. Pediatr. Gastroenterol. Nutr. 2008, 47, 45-53.

66. Mohan, R.; Koebnick, C.; Schildt, J.; Mueller, M.; Radke, M.; Blaut, M. Effects of Bifidobacterium lactis Bb12 supplementation on body weight, fecal $\mathrm{pH}$, acetate, lactate, calprotectin, and IgA in preterm infants. Pediatr. Res. 2008, 64, 418-422.

67. Ivory, K.; Chambers, S.J.; Pin, C.; Prieto, E.; Arqués, J.L.; Nicoletti, C. Oral delivery of Lactobacillus casei Shirota modifies allergen-induced immune responses in allergic rhinitis. Clin. Exp. Allergy 2008, 38, 1282-1289.

68. Htwe, K.; Yee, K.S.; Tin, M.; Vandenplas, Y. Effect of Saccharomyces boulardii in the treatment of acute watery diarrhea in Myanmar children: A randomized controlled study. Am. J. Trop. Med. Hyg. 2008, 78, 214-216.

69. Larsson, P.G.; Stray-Pedersen, B.; Ryttig, K.R.; Larsen, S. Human lactobacilli as supplementation of clindamycin to patients with bacterial vaginosis reduce the recurrence rate; a 6-month, double-blind, randomized, placebo-controlled study. BMC Womens Health 2008, 8, 3.

70. Hatakka, K.; Ahola, A.J.; Yli-Knuuttila, H.; Richardson, M.; Poussa, T.; Meurman, J.H.; Korpela, R. Probiotics reduce the prevalence of oral candida in the elderly-A randomized controlled trial. J. Dent. Res. 2007, 86, 125-130. 
71. Basu, S.; Chatterjee, M.; Ganguly, S.; Chandra, P.K. Effect of Lactobacillus rhamnosus GG in persistent diarrhea in Indian children: A randomized controlled trial. J. Clin. Gastroenterol. 2007, $41,756-760$.

72. Henker, J.; Laass, M.; Blokhin, B.M.; Bolbot, Y.K.; Maydannik, V.G.; Elze, M.; Wolff, C.; Schulze, J. The probiotic Escherichia coli strain Nissle 1917 (EcN) stops acute diarrhoea in infants and toddlers. Eur. J. Pediatr. 2007, 166, 311-318.

73. Sugawara, G.; Nagino, M.; Nishio, H.; Ebata, T.; Takagi, K.; Asahara, T.; Nomoto, K.; Nimura, Y. Perioperative synbiotic treatment to prevent postoperative infectious complications in biliary cancer surgery: A randomized controlled trial. Ann. Surg. 2006, 244, 706-714.

74. Krasse, P.; Carlsson, B.; Dahl, C.; Paulsson, A.; Nilsson, A.; Sinkiewicz, G. Decreased gum bleeding and reduced gingivitis by the probiotic Lactobacillus reuteri. Swed. Dent. J. 2006, 30, $55-60$.

75. Margreiter, M.; Ludl, K.; Phleps, W.; Kaehler, S.T. Therapeutic value of a Lactobacillus gasseri and Bifidobacterium longum fixed bacterium combination in acute diarrhea: A randomized, double-blind, controlled clinical trial. Int. J. Clin. Pharmacol. Ther. 2006, 44, 207-215.

76. Olivares, M.; Díaz-Ropero, M.A.; Gómez, N.; Lara-Villoslada, F.; Sierra, S.; Maldonado, J.A.; Martín, R.; López-Huertas, E.; Rodríguez, J.M.; Xaus, J. Oral administration of two probiotic strains, Lactobacillus gasseri CECT5714 and Lactobacillus coryniformis CECT5711, enhances the intestinal function of healthy adults. Int. J. Food Microbiol. 2006, 107, 104-111.

77. Sarker, S.A.; Sultana, S.; Fuchs, G.J.; Alam, N.H.; Azim, T.; Brüssow, H.; Hammarström, L. Lactobacillus paracasei strain ST11 has no effect on rotavirus but ameliorates the outcome of nonrotavirus diarrhea in children from Bangladesh. Pediatrics 2005, 116, e221-e228.

78. Schrezenmeir, J.; Heller, K.; McCue, M.; Llamas, C.; Lam, W.; Burow, H.; Kindling-Rohracker, M.; Fischer, W.; Sengespeik, H.C.; Comer, G.M.; et al. Benefits of oral supplementation with and without synbiotics in young children with acute bacterial infections. Clin. Pediatr. (Phila.) 2004, 43, 239-249.

79. Reid, G.; Burton, J.; Hammond, J.A.; Bruce, A.W. Nucleic acid-based diagnosis of bacterial vaginosis and improved management using probiotic lactobacilli. J. Med. Food. 2004, 7, 223-228.

80. Morelli, L.; Zonenenschain, D.; Del Piano, M.; Cognein, P. Utilization of the intestinal tract as a delivery system for urogenital probiotics. J. Clin. Gastroenterol. 2004, 38, S107-S110.

81. Reid, G.; Charbonneau, D.; Erb, J.; Kochanowski, B.; Beuerman, D.; Poehner, R.; Bruce, A.W. Oral use of Lactobacillus rhamnosus GR-1 and L. fermentum RC-14 significantly alters vaginal flora: Randomized, placebo-controlled trial in 64 healthy women. FEMS Immunol. Med. Microbiol. 2003, 35, 131-134.

82. Arvola, T.; Laiho, K.; Torkkeli, S.; Mykkänen, H.; Salminen, S.; Maunula, L.; Isolauri, E. Prophylactic Lactobacillus GG reduces antibiotic-associated diarrhea in children with respiratory infections: A randomized study. Pediatrics 1999, 104, e64.

83. Schulz, K.F.; Altman, D.G.; Moher, D.; CONSORT Group. CONSORT 2010 statement: Updated guidelines for reporting parallel group randomised trials. J. Clin. Epidemiol. 2010, 63, 834-840.

84. Montané, E.; Vallano, A.; Vidal, X.; Aguilera, C.; Laporte, J.R. Reporting randomised clinical trials of analgesics after traumatic or orthopaedic surgery is inadequate: A systematic review. BMC Clin. Pharmacol. 2010, 10, 2. 
85. Thomson Reuters. Available on line: http://www.thomsonreuters.com/products_services/science/ science_products/a-z/journal_citation_reports/(accessed on 14 May 2013).

86. Teughels, W.; van Essche, M.; Sliepen, I.; Quirynen, M. Probiotics and oral healthcare. Periodontol. 2000 2008, 48, 111-147.

87. Campus, G.; Cagetti, M.G.; Sale, S.; Petruzzi, M.; Solinas, G.; Strohmenger, L.; Lingström, P. Six months of high-dose xylitol in high-risk caries subjects-A 2-year randomised, clinical trial. Clin. Oral Investig. 2013, 17, 785-791.

88. EFSA Panel on Dietetic Products, Nutrition and Allergies. Scientific Opinion on the substantiation of health claims related to Lactobacillus reuteri ATCC 55730 and "natural defence" (ID 905) pursuant to Article 13(1) of Regulation (EC) No 1924/2006. EFSA J. 2010, 8, 1805.

89. EFSA Panel on Dietetic Products, Nutrition and Allergies. Scientific Opinion on the substantiation of health claims related to Lactobacillus rhamnosus ATCC 53103 (LGG) and "gastro-intestinal health" (ID 906) and maintenance of tooth mineralisation (ID 3018) pursuant to Article 13(1) of Regulation (EC) No 1924/2006. EFSA J. 2011, 9, 2233.

90. Bath, F.J.; Owen, V.E.; Bath, P.M. Quality of full and final publications reporting acute stroke trials. A systematic review. Stroke 1998, 29, 2203-2210.

91. Garfield, E. Journal impact factor: A brief review. Can. Med. Assoc. J. 1999, 161, 979-980.

(C) 2013 by the authors; licensee MDPI, Basel, Switzerland. This article is an open access article distributed under the terms and conditions of the Creative Commons Attribution license (http://creativecommons.org/licenses/by/3.0/). 